\title{
Globe
}

Revue internationale d'études québécoises

\section{La formation générale en tant que projet critique et herméneutique}

\section{Louis LeVasseur}

Volume 1, numéro 2, 1998

URI : https://id.erudit.org/iderudit/1000511ar

DOI : https://doi.org/10.7202/1000511ar

Aller au sommaire du numéro

Éditeur(s)

Globe, Revue internationale d'études québécoises

ISSN

1481-5869 (imprimé)

1923-8231 (numérique)

Découvrir la revue

Citer cet article

LeVasseur, L. (1998). La formation générale en tant que projet critique et herméneutique. Globe, 1(2), 29-43. https://doi.org/10.7202/1000511ar
Résumé de l'article

Les cours de français et de philosophie sont généralement conçus comme étant les axes fondamentaux de la formation générale. Cependant, les contenus de ces cours se transforment au fil des années, ainsi que leur légitimation pédagogique et sociale. Dans cet article, l'auteur donne des indications de l'évolution du cours collégial de philosophie de 1961 à 1995, et il définit un projet de formation générale lié à des intérêts critique et herméneutique. d'utilisation que vous pouvez consulter en ligne.

https://apropos.erudit.org/fr/usagers/politique-dutilisation/ 


\title{
La formation générale en tant que projet critique et herméneutique
}

\author{
Louis LeVasseur \\ Université de Montréal
}

Il existe depuis au moins une quinzaine d'années, dans les sociétés industrielles avancées, un discours selon lequel l'éducation doit être un sous-système du système économique. Par conséquent, sa fonction critique devrait être abandonnée, ou du moins, demeurer subsidiaire à sa fonction économique 1 . Ce discours est bien évidemment sur les lèvres de bon nombre de directeurs d'entreprises, de tous les tenants du néolibéralisme et de plusieurs hommes d'État. Mais il est également entériné par le ministère de l'Éducation et les directeurs d'établissements d'enseignement.

Par ailleurs, certaines pratiques intellectuelles s'inscrivant dans des "paradigmes à caractère formaliste», dont l'analyse des discours, ont gagné en force au cours des trente demières années dans les établissements d'enseignement supérieur du Québec. Ces courants intellectuels se caractérisent par un renoncement

1 ROCHER, Guy, «Re-définition du rôle de l'université», L'Éducation 25 ans plus tard! Et après?, sous la direction de Fernand Dumont et de Yves Martin, Québec, Institut québécois de recherche sur la culture, pp. 181-198.

Louis LeVasseur, "La formation générale en tant que projet critique et

herméneutiquen, Globe. Revue internationale d"ítudes québécoises, vol. 1, no 2, 1998. 
explicite à toute prise en compte de la dimension subjective de l'action humaines?

La présence des "paradigmes à caractère formaliste» et celle du discours économique ont considérablement modifié la définition de la formation générale au niveau collégial. Celle-ci a pris un tournant objectiviste et, dans une moindre mesure, un tournant techniciste dont nous donnerons un aperçu à travers les débats portant sur l'enseignement collégial de la philosophie. Mais nous chercherons surtout, dans cet article, à déterminer la fonction de la formation générale dans le contexte de la modemité tardive. Doit-elle répondre ̀̀ un intérêt herméneutique ou critique? Le fait que certaines de ses composantes soient déjà arrimées à un projet technique compromet-il les fonctions herméneutique et critique de la formation générale?

\section{La formation générale en tant que projet critique}

La formation générale est habituellement définie par opposition à la formation spécialisée. Celle-ci doit permettre à l'étudiant d'acquérir les savoirs et de développer les compétences qui lui permettront de s'insérer dans le marché du travail. La formation générale répond à d'autres besoins éducatifs par des savoirs différents des savoirs spécialisés et techniques. Ces besoins diffèrent selon les sociétés et selon les époques. La formation générale n'est donc pas un concept abstrait et invariable. Elle prend différentes formes dans la réalité historique de l'enseignement.

2 FREITAG, Michel, «La crise des sciences sociales et la question de la normativité", Le Naufrage de l'université, Québec, Nuit Blanche éditeur et Paris, Éditions de la découverte, 1995, p. 76. 
D'ailleurs, depuis la création des cégeps, la formation générale a évolué. Il suffit de jeter un coup d'œil sur les programmes d'enseignement pour le constater. Notre attention portera sur les transformations du programme collégial de philosophie ${ }^{3}$, lesquelles se rapportent à l'objet de la critique qu'élabore la philosophie collégiale, à l'espace occupé par les activités critiques dans le programme et à la légitimation du programme.

Définissons d'abord l'objet de la critique philosophique. Dans les années 70, cet objet était constitué par le milieu socioculturel de l'étudiant, les manifestations de pouvoir dans les domaines politique, social et culturel. Dans les années 80 et 90 , la critique a pris la forme d'une analyse des discours des systèmes de pensée philosophiques ou les représentations culturelles dans l'histoire. Par exemple, au lieu de critiquer les valeurs culturelles ambiantes et de s'engager dans une réflexion de type existentialiste, l'étudiant devait parvenir à une connaissance objective de la philosophie existentialiste, ou encore de représentations de la nature ou de «systèmes éthiques et politiques». Autrement dit, la critique de la culture contemporaine a cédé le pas à une étude théorique (ou conceptuelle) et historique des courants de pensée.

Il n'y a pas que l'objet de la critique qui ait changé au cours des années, il y a également l'espace occupé par la critique dans le programme de philosophie. Ceci est tout à fait représentatif du changement de rapport à la culture dans la philosophie collégiale, et d'une manière plus générale, dans la modernité tardive. Expliquons. Ce qui caractérisait au premier chef la philosophie des premiers régimes pédagogiques (ceux de 1967 à

3 La philosophie constituait, avec le français et l'éducation physique, l'un des trois axes de la formation générale obligatoire pour tous les étudiants du collégial de 1967 à 1995 . Depuis 1995, l'anglais fait partie du programme de formation générale. 
1973), c'était son articulation par rapport aux projets d'appropriation et de distanciation. L'appropriation doit être comprise comme étant l'activité par laquelle l'étudiant cherchait à "s'appropries la tradition culturelle à laquelle il appartenait, ou encore cherchait à s'investir dans son propre milieu socioculturel afin de le redéfinir, de le transformer, de se l'«approprien. L'interprétation de l'héritage culturel d'une société et la redéfinition des valeurs par l'étudiant à la lumière de cet héritage de sens sont des exemples d'appropriationt. Quant à la distanciation, elle doit être comprise comme étant la critique non seulement de la tradition, mais également de la modemité, des institutions sociales, du pouvoir politique et du pouvoir culturels. Or, les projets d'appropriation et de distanciation ne figuraient plus dans les programmes ministériels à partir de 1977. De plus, avec l'adoption, par la Coordination de la philosophie collégiale en 1992, d'une séquence d'enseignement axée sur le développement des "habiletés intellectuelles», la critique figurait désormais à côté de cinq autres "habiletés»"

4 PROULX, Jean, «Enseignement de la philosophie et objectifs de culture générale", Actes du colloque sur l'enseignement de la philosophie, Montréal, Université du Québec à Montréal, département de philosophie, juin 1973, p. 56.

5 Femand Dumont a fait référence à la «faculté d'oppression que constitue l'usage de la parole, de la science, des savoirs, de l'idéologie, qui prennent une place déterminante dans nos sociétés». "Sociologie et sociologues québécois. Fernand Dumont), (enregistrement vidéo), Service des ressources pédagogiques, Université Laval, 1981. Cette référence à Dumont n'est pas fortuite. Sa pensée a largement servi de ferment à la définition du programme de philosophie de 1973 dans lequel apparaissent les concepts d'appropriation et de distanciation. Dans les documents pédagogiques de l'époque afférents à la philosophie collégiale, les travaux de Dumont étaient fréquemment cités.

6 Les «habiletés intellectuelles» sont l'équivalent de compétences intellectuelles ou génériques. Elles sont généralement associées à des capacités de langage, de raisonnement, d'argumentation. 
conceptualiser, analyser, comparer, problématiser et synthétiser. En regard de cette séquence d'enseignement, les activités critiques ne semblent occuper qu'un espace périphérique dans les programmes. Toutefois, et ceci semble paradoxal, la critique, par rapport à celle des années 70 , s'est hypertrophiée dans les années 90. C'est que la critique, comme nous l'avons vu, a désormais un statut épistémologique et non plus social, culturel ou politique. La critique a pour objet les représentations du monde dans l'histoire, et moins les phénomènes socioculturels contemporains. En prenant pour objet les représentations du monde et les systèmes de pensée, l'étudiant est amené à évaluer la validité de leurs fondements et à les considérer comme étant relatifs et contingents. Mais comment peut-il, à la suite de cette suspicion systématique à leur endroit, s'investir en l'un d'eux afin de définir pour son propre compte le sens du monde? La critique dans la philosophie collégiale semble donc correspondre à un travail de la culture que Femand Dumont identifie à un "retour sur les genèses», travail dont il montre le caractère antinomique avec le «travail de mémoire»?

Voyons maintenant en quoi les modes de légitimation de l'enseignement de la philosophie ont changé des années 70 aux années 90. En 1975, la Coordination de la philosophie collégiale pourfendait le Conseil du patronat pour sa conception utilitariste de l'éducation et son apologie du capitalisme. La Coordination soutenait que le rôle de la philosophie consistait en un éveil des consciences des étudiants aux réalités socioculturelles, par exemple les phénomènes de domination sociale. En 1992, aux audiences parlementaires sur la réforme de l'enseignement collégial, la question de la pertinence du cours de philosophie a été examinée. La stratégie de la Coordination ${ }^{8}$

7 DUMONT, Fernand, L'Anthropologie en l'absence de l'bomme, Paris, Presses universitaires de France, 1981, p. 203.

8 «Le rôle de la philosophie dans le renouveau du DEC», Mémoire présenté le 28 septembre 1992 à la Commission de l'éducation 
consistait à montrer que l'enseignement de la philosophie favorisait le développement de compétences intellectuelles" lesquelles permettaient à l'étudiant de s'adapter aux nouveaux besoins de l'économie dans un contexte de mondialisation des marchés. Dans les années 70, la philosophie collégiale dictait ses exigences au milieu culturel et économique; dans les années 90 , elle cherchait plutôt à s'y adapter. Nous ne voulons aucunement signifier ici que les objectifs de la philosophie collégiale se confondent intégralement avec les impératifs de la production matérielle et de la gestion technocratique du social, mais souligner que la nature du discours de la Coordination de 1992 est peut-être indicative d'une amorce de dérive vers un intérêt technique.

Nous venons d'évoquer l'évolution du statut de la critique dans la philosophie collégiale et nous avons même donné des indications quant à sa place, en tant qu'institution, dans la culture et dans la société. Notre position par rapport à la critique est la suivante. Il importe que la possibilité soit donnée à l'étudiant de se situer dans son propre univers culturel et de déceler les intérêts et les enjeux de pouvoir qui apparaissent dans les discours idéologiques et institutionnels. En ce sens, la critique dans un programme de culture générale serait beaucoup plus orientée vers l'acquisition de dispositions culturelles que vers le développement de compétences intellectuelles, ou vers

chargée de faire le point sur l'enseignement collégial québécois par Jean-Marie Therrien et Pierre Cohen-Bacrie, pour «Philosophie au Collège». Le texte a été reproduit dans la revue Pbilosopher, no 13, 1992, pp. 11-46. Il est à noter que M. Cohen-Bacrie était Coordonnateur québécois de la philosophie collégiale en 1992.

9 Le mémoire fait plutôt référence à des «habiletés intellectuelles», lesquelles sont définies comme étant des "aptitudes requises pour produire un discours rationnel argumentatif tel que la dissertation philosophique». Voir : «Le rôle de la philosophie dans le renouveau du DEC), op. cit., p. 46. 
une instrumentalisation de la pensée vers laquelle semble conduire la séquence d'enseignement axée sur le développement de ces compétences. Ces dispositions culturelles doivent impérativement permettre à l'étudiant de s'engager dans des activités herméneutiques et critiques, de nommer l'être après avoir libéré celui-ci de ses significations les plus contraignantes et discordantes. Examinons donc ces activités d'appropriation, ou herméneutiques, par lesquelles l'étudiant parvient à donner un sens à son univers, à son existence.

\section{La formation générale en tant que projet herméneutique}

Voyons à quoi correspondrait, théoriquement, un programme de formation générale axé sur un intérêt pratique ou herméneutique. Notre attention portera d'abord sur l'herméneutique interprétative du philosophe allemand HansGeorg Gadamer et sur les conceptions de l'histoire et de la vérité qui y sont développées.

Gadamer considère l'histoire comme étant le véhicule d'un sens qui présente un intérêt pratique ou éthique pour l'interprète. Son projet herméneutique est dirigé en bonne partie contre la vérité de type méthodologique développée dans les sciences historiographiques. Comprendre, selon lui, ce n'est pas analyser, reconstituer objectivement le climat intellectuel d'une époque, mais bien appliquer à soi-même un sens qui provient de la tradition. Le dialogue doit être constant entre le présent et le passé, ce qui signifie qu'il doit être motivé par un intérêt pratique. L'interprète qui se met à l'écoute de la tradition en reçoit un sens qu'il applique à sa propre situation dans le 
monde ${ }^{10}$. Il n'y a donc pas de coupure temporelle, dans le sens d'une mise à distance, dans la conception gadamérienne de l'histoire, mais une transmission de sens qui nourrit la conscience de l'être-là. L'analyse positiviste de l'histoire favorise la mise à distance qui sépare le chercheur du contexte étudié, car celle-ci constitue, d'un point de vue méthodologique, un gage d'objectivité. L'herméneutique gadamérienne cherche coûte que coûte à abolir cette mise à distance pour que le passé soit rendu aussi présent que possible:

Le texte compris en historien est formellement dépossédé de la prétention à dire quelque chose de vrai. Lorsque l'on considère la tradition en historien, que l'on

10 L'acte de comprendre, d'interpréter, n'est aucunement un acte de soumission face à l'histoire. Si le passé a un effet décisif sur le présent, ce que Gadamer appelle l'chistoire de l'efficiencel, l'interprète agit en retour sur le passé, le comprend toujours différemment selon la situation et le moment où il se trouve. Il n'est donc pas soumis entièrement à son déterminisme. Les préoccupations de l'interprète modifient le regard qu'il porte sur le passé. L'histoire est mouvante et ne cesse d'offrir des occasions nouvelles d'interprétation. Toutefois, comme le souligne Jean Grondin, la compréhension n'est pas un «agir de la subjectivité autonome» : «a compréhension, ou ce qui signifie dorénavant la même chose, l'application est moins un agir de la subjectivité autonome que le fait d'une "insertion dans un événement de tradition où se médiatisent constamment le passé et le présent". Comprendre un texte du passé, c'est savoir le traduire pour notre situation présente, entendre en lui un écho aux inquiétudes de notre temps. Cette "contemporanéité" des textes anciens se manifeste déjà dans le canon des œuvres qui s'est imposé à notre actualité. Pourquoi retenir telle cuvre du passé plutôt que telle autre sinon parce qu'elle est plus parlante, plus déterminante pour notre histoire et, partant, pour notre compréhension de nous-mêmes?» L'Universalité de l'berméneutique, Épiméthée, Paris, Presses universitaires de France, 1993, p. 177. 
se replace dans la situation historique et que l'on cherche à reconstituer l'horizon historique, on croit comprendre. En réalité, on a fondamentalement renoncé à l'ambition de trouver dans la tradition une vérité qui elle-même s'imposerait et se ferait comprendre. Cette manière de reconnaitre l'altérité de l'autre, qui en fait un objet de connaissance objective, équivaut à suspendre dans le principe sa prétention. ${ }^{11}$

Pour Gadamer, deux attitudes sont possibles devant le texte. La première, qu'il juge insatisfaisante, consiste à déterminer la spécificité stylistique d'un texte, ou encore à définir la pensée de l'auteur, à découvrir ses intentions en recourant à une analyse générique, c'est-à-dire en faisant intervenir des éléments extérieurs au texte pour l'expliquer, comme la biographie et la psychologie de l'auteur, le contexte social d'où l'œuvre est issue. La seconde consiste à considérer le texte comme ayant quelque chose à nous dire ou à nous révéler sur nous-mêmes et sur notre situation historique.

Ainsi, d'après Gadamer, l'œuvre artistique ou littéraire qui se trouve là, devant nous, n'est pas que belle. Elle est vraie dans' sa signification et dans l'exigence qu'elle nous impose. La présence de l'cuvre a le pouvoir de nous subjuguer, de nous irradier, de nous révéler à nous-mêmes. C'est en ce sens que le passé est présent et qu'il est source de connaissance. Le concept d'application de Gadamer suppose donc que l'expérience du passé conserve son efficace dans le présent. Cette compréhension n'est pas à concevoir dans le sens d'une analyse se traduisant par des résultats objectivables, mais dans le sens d'une expérience devant servir à mieux nous tirer d'affaire dans le monde et à répondre à nos inquiétudes du moment. $\mathrm{La}$ connaissance herméneutique

11 GADAMER, Hans-Georg, Vérité et méthode, édition intégrale revue et complétée par Pierre Fruchon, Jean Grondin et Gilbert Merlio, Paris, Éditions du Seuil, 1976, p. 325. 
est un savoir d'expérience qui a peu en commun avec le savoir scientifique. Dans l'extrait suivant, Gadamer montre comment l'application s'exerce dans les domaines juridique et religieux :

Une loi ne demande pas à être comprise historiquement, mais doit se concrétiser dans sa valeur juridique à travers l'interprétation. De même, un texte religieux ne demande pas à être considéré comme un simple document historique, il doit être compris de façon à exercer son action salvatrice. Comprendre le texte de façon adéquate, c'est-à-dire conformément à son ambition, implique dans les deux cas l'obligation de comprendre le texte, qu'il soit loi ou message de salut, de façon nouvelle et différente à chaque instant, c'est-à-dire dans chaque situation concrète. Ici, comprendre, c'est déjà appliquer. ${ }^{12}$

La tradition permet donc de répondre au questionnement existentiel de l'être-là mais, en même temps, elle permet à ce demier de mettre à l'épreuve ses préjugés, de revoir sa propre structure pré-conceptuelle. La confrontation avec les œuvres de la tradition est l'occasion de prendre conscience des anticipations qui le guident et de leurs limites, et ce faisant, d'accéder à des vérités $^{13}$. C'est en ce sens que Gadamer soutient que les œuvres artistiques et littéraires ne sont pas que belles. Elles contiennent des éléments de connaissance qui trouvent leur application non pas dans le domaine technique mais pratique. Ainsi, la littérature ne serait pas limitée à la sphère de l'esthétique. Mais en quoi la littérature permet-elle la confrontation avec le lecteur, en quoi transcende-t-elle les limites de la beauté formelle et l'expérience purement esthétique?

12 GADAMER, Hans-Georg, op. cit., p. 150.

13 GADAMER, Hans-Georg, op. cit., p. 20. 
Dans un article intitulé "Le fantôme de la littérature», Jean Larose a montré les vertus pédagogiques de la littérature. Pour cela, il interprétait le mot de Diderot dans le Paradoxe sur le comédien selon lequel le comédien est un "spectateur froid». Si le comédien est spectateur, se demande Jean Larose, de quoi l'est-il donc?

[...] le grand comédien est spectateur - de la transformation prodigieuse qui l'élève au-dessus de huimême quand il se laisse posséder, de façon démoniaque, par un "grand fantôme» qu'il s'est fabriqué, et qui est l'idée qu'il se fait de son personnage, Phèdre, Agamemnon ou Ophélie. «Mais alors, écrit Diderot, ce n'est plus lui qui agit, c'est l'esprit d'un autre qui le domine». Le grand homme est ainsi celui qui sait se laisser dominer, comme par le géant de lui-même, lequel est un autre qui en sait plus que hui, qui voit plus loin que lui, qui ressortit à un monde entièrement fabriqué, et dont l'appartenance à ce registre artificiel est la condition même de sa vérité.

Voilà qui exprime pour moi le sens méconnu de l'enseignement de la littérature. Quand Diderot déclare au sujet du comédien : "c'est l'esprit d'un autre qui le domines, cela décrit aussi la formation que donne la littérature, le miracle éducatif opéré par des textes dont toute l'efficacité formative repose sur cette fausseté, sur cette artificialité - que leur reprochent les pédagogues. ${ }^{14}$

La littérature dont parle Jean Larose ici met en œuvre une vérité non pas méthodologique ni objective, mais pratique ou herméneutique. Il évoque d'ailleurs le fait que ce type de culture n'a pas la faveur des pédagogues. En fait, depuis trente ans au Québec, parler de vérité dans une discipline autre que scientifique

14 LAROSE, Jean, L'Amour du pauvre, Montréal, Boréal, 1991, p. 23. 
rend suspect. De plus, l'idée que les cuvres maîtresses de la littérature puissent conduire à faire l'expérience de la vérité éveille des soupçons d'élitisme, sinon de dogmatisme ${ }^{15}$. Or, c'est précisément cette méfiance envers la vérité des systèmes de pensée qui anime la philosophie collégiale depuis les années 80 . L'étudiant est amené à se dresser devant l'esprit de l'autre, même si la domination dont il est question chez Larose, chez Diderot et même chez Gadamer est salvatrice ou émancipatrice.

Mais revenons à notre problématique de départ : quels fondements théoriques la culture générale doit-elle avoir? Le contexte de la modernité tardive, que caractérise entre autres la domination de la rationalité scientifique et technocratique, rend nécessaire une instance critique. Mais une instance pratique ou herméneutique, telle que nous venons de la définir, doit prendre le relais de la critique afin de favoriser les reconstructions de sens. À cet égard, nous venons de le démontrer, la littérature semble tout indiquée.

Mais compte tenu des pressions qu'exercent les milieux des affaires et les États sur nos systèmes d'éducation afin que ceux-ci contribuent activement à relever les défis de la mondialisation des marchés et de la concurrence économique, et compte tenu également de la volonté des étudiants de recevoir une formation leur permettant de s'insérer le plus adéquatement possible dans le marché de l'emploi ${ }^{16}$, les savoirs critiques et herméneutiques semblent inutiles. Ce qui ne signifie aucunement qu'ils sont

$15 \mathrm{Ni}$ Gadamer ni Larose ne défendent dans leur œuvre une conception absolutiste de la vérité. Celle-ci y est plutôt conçue dans une perspective de finitude humaine. En revanche, il faut bien se garder de les ranger dans une catégorie d'intellectuels qui défendent un relativisme culturel selon lequel toutes les idées et les cuvres de la pensée seraient d'égale valeur.

16 ROCHER, Guy, op. cit., pp. 181-198. 
sans pertinence. Mais de quelle pertinence s'agit-il ici? Elle est double : sociale et individuelle.

La pertinence sociale de ces savoirs peut être illustrée par une anecdote que racontait Femand Dumont dans l'un de ses articles. À l'occasion d'une séance de réflexion sur l'enseignement de la philosophie, où chaque participant passait individuellement en entrevue, Dumont fut invité à commenter l'affirmation d'un ingénieur selon laquelle on n'avait pas besoin de philosophes pour construire un réfrigérateur, autrement dit, pour améliorer les performances du système de production. $\mathrm{Ce}$ à quoi Fernand Dumont acquiesça, en ajoutant même que nous avions besoin de réfrigérateurs et de l'art de les construire. Mais il affirmait également que c'est par la philosophie qu'il était possible de proclamer le "dépassement de l'homme» et celui du «besoin»"

Quant à la pertinence individuelle de ces savoirs, elle n'est pas à chercher dans d'éventuels avantages matériels et professionnels qu'ils permettraient d'obtenir, ou dans le fait qu'ils rendent le travailleur plus performant. Ce serait là une manière de donner une légitimation technique à ces savoirs et de les inféoder aux impératifs économiques. Leur pertinence individuelle est plutôt dans la possibilité qu'ils présentent de raffiner notre vision des choses, de prendre conscience de nos déterminations, de parvenir à une conversion du regard. Ce sont là les dures conditions de l'enrichissement de nos vies personnelles ${ }^{18}$ mais également, par conséquence, de l'enrichissement de notre avenir collectif.

Or, les partisans de l'objectivisme philosophique et ceux des théories constructivistes en pédagogie soutiennent qu'il n'y a pas

17 DUMONT, Fernand, "Urgence et tradition de la philosophie», Le Sort de la culture, Montréal, L'Hexagone, 1987, pp. 220-223.

18 Pensons à la douloureuse sortie de la caveme chez Platon. 
d'incompatibilité entre la mise à distance des systèmes de pensée (activités critiques) et la construction du sens par l'élève (activités herméneutiques). Ce demier serait libre, après la mise en extériorité des systèmes de pensée, d'adhérer au système qui lui semble le mieux fondé. Qui plus est, la présentation objective des représentations du monde et des systèmes de pensée préviendrait les excès doctrinaires et dogmatiques. Il convient de reconnaitre, à ce chapitre, la valeur de ce type de formation. Mais trois remarques s'imposent ici. La fonction de la connaissance objective n'est pas d'abord de rendre le monde plus significatif pour le sujet. La découverte des principes qui sont au fondement du réel contreviennent à la manière dont le sujet habite le monde. Deuxièmement, la construction du sens par le sujet à partir de ses propres ressources, donc sans la médiation de la tradition, se heurte bien souvent à la réalité sociale. Autrement dit, l'idéalisme subjectif est impuissant à saisir les contradictions qui sont au fondement du réel historique et la manière dont les pouvoirs politiques, sociaux et culturels déterminent notre conscience du monde. Troisièmement, la tradition et la mémoire ne doivent pas être perçues exchusivement comme étant constituées de significations premières du monde que la connaissance scientifique prend pour objet. Elles contiennent également des références qui permettent de procéder à un retour réflexif sur ces significations premières du monde, mais selon des modalités qui ne sont pas celles des sciences méthodologiques ou de l'analyse du discours. Et c'est précisément cette dimension réflexive inscrite dans une perspective normative qui est absente actuellement de nos étblissements d'enseignement.

Ces remarques étant faites, un choix demeure. Celui d'associer la formation générale à un projet objectif de la culture ou à un projet normatif de la culture. Le premier projet en est un de vérité. Le second en est un de pertinence et il implique la transmission de la culture. C'est sans compter que certains optent plutôt pour une voie beaucoup plus pragmatique où la 
REVUE INTERNATIONALE D'ÉTUDES QUÉBÉCOISES

formation génémle viserait à développer les compétences nécessaires à l'intégration à un monde du travail de plus en plus technologique. 